MATEC Web of Conferences 34, 05001 (2015)

DOI: $10.1051 /$ matecconf/ 20153405001

(c) Owned by the authors, published by EDP Sciences, 2015

\title{
The Mechanism Study of Alternating Arc(AC)Magnetic Levitation Induction Motor
}

\author{
Zeng $\mathrm{Li}^{1, a^{*}}$, Zhang Fan ${ }^{1, \mathrm{~b}}$, Zhu Zhida ${ }^{1, c}$, Chen Fang ${ }^{1, a}$ \\ ${ }^{1}$ College of Mechanical Engineering, Yangzhou University, Yangzhou 225127, China
}

\begin{abstract}
Magnetic levitation (no bearings) motor by using magnetic force to make rotor suspend and drive realize its high or ultra-high speed rotating. The stator's structure of traditional no bearing magnetic levitation motor is double winding which is polar logarithmic difference 1 of 2 sets of winding (torque winding and suspension winding) and embedded in the stator. Using two inverter respectively for the two sets of winding to go into the same frequency current in order to realize the suspension of the rotor and motor's driven, small carrying capacity of motor's structure, controlling complex system. This paper based on the traditional motor technology puts forward a kind of arc principle and respectively decorates two arc motors in horizontal and vertical direction symmetric to rotor according to the electromagnetic bearing suspension technology that is constituted the arc magnetic levitation induction motor. Establishing air-gap transformation regular between rotor and stator (air-gap length) motor is under the effect of interference. Based on the electromagnetic theory establishing distribution regular of the air-gap magnetic induction intensity. Virtual displacement principle is used to establish electromagnetism mathematical model and motor electromagnetism levitation. By the finite element analysis carrying on simulation research to the magnetic induction intensity, electric magnetic levitation force and distribution features of electromagnetic torque and so on.
\end{abstract}

\section{Introduction}

Magnetic levitation motor superposes winding producing radial force in the electromagnetic bearing on the motor's stator winding, which produces two different logarithmic rotating magnetic fields in the air gap, so as to produce torque and levitation force at the same time, in order to make the motor drive and the suspension of the rotor [1]. Magnetic levitation motor stator has double and single windings of these two kinds of winding structure. At present, most of the magnetic levitation motor stator is double winding structure, that is embedded 2 sets of winding that logarithmic difference is 1 (torque winding and suspension winding) in the stator pole, and two inverters respectively give the two sets of winding the same frequency current so as to realize the motor drive and the rotor suspension, but this kind of motor structure have small bearing capacity and complex control system. For this, this paper come up with a kind of a new motor of Arc magnetic levitation induction motor, which is based on 4 lengths of arc stators. New type of magnetic suspension motor winding structure and the traditional motor stator winding structure are the same. It gets a

Corresponding author: ${ }^{\mathrm{a}}$ lizengcf@ 163. .com;

b fzhang@yzu.edu.cn, , ${ }^{\mathrm{b}}$ zdzhu@yzu.edu.cn balance of magnetic fields by changing stator winding of the alternating arc motor and so as to produce levitation force for the rotor levitation ${ }^{[1-4]}$. Compared with ordinary double-winding magnetic suspension motor, ac arc magnetic levitation induction motor has simple structure, small motor power loss ${ }^{[5]}$. Aiming at an arc magnetic levitation induction motor, this paper studies the rotation and suspension mechanism, and establishes the mathematical model of the motor system, analyzes the characteristics of suspension and rotation of the motor by means of finite element method.

\section{The mechanism of alternating ac mag- netic induction motor}

Alternating arc magnetic levitation induction motor employs four 3-phase alternating arc motors to make rotor suspend and drive it to rotate. Based on the traditional motor technology, it rolls windings on the open arc stator and then applies electric current to make it rotate. Arc motor is analogous to the traditional ones, since it can be classified as 3-phase motor, 2-phase motor 
or single-phase motor and other alternating (synchronous or asynchronous) motors. What's more there are also direct motor, stepping (or reluctance) motor, etc. The stator winding of alternating arc motor can generate a rotating magnetic field, cut the rotor closed loop winding and it has to be a donating current and potential so that the rotor shaft may rotate at the same time.

Alternating arc magnetic levitation induction motor consists of stator, rotor and levitation/rotation controlling test system and so on. The motor performs on the basis of electromagnetic bearing suspension technology, compared with the stator core of traditional motor. There are two 3-phase alternating arc motor windings in horizontal and vertical directions symmetric with rotor respectively. With the effect of comprehensive controlling test system, the motor can differentially adjust the increase current changes of the arc motor on both sides in a direction of the rotor, in order to change the magnetic pull applied to rotor from 2-arc motor, which make the rotor continuously work in the direction of equilibrium position at the center of the 2-arc motor.

In Fig. 1, it shows Y-type connection of each threephase winding and its electrifying state at a certain moment. 4 parts of arc motor's stator winding are respectively and symmetrically placed 2 arc in the direction of the $X$ axis and $Y$ axis. Through the parallel connection of 3 phase alternating-current $i_{1}, i_{2}, i_{3}$ to 4 parts of arc motor's three-phase stator winding so as to separately control current amplitude of each arc motor's stator winding in manners of Closed loop, then realize the adjustment of electromagnetic magnetic pull to rotor and generate controllable electromagnetism levitation. The principle of Rotor suspension is equal to the Mechanisms of traditional electromagnetic bearing ${ }^{[6,7]}$, in the effect of interference, the rotor shifts $\Delta x$ along forward (right) direction of $X$ axis, in order to make the rotor return to the center of the stator (equilibrium position) and work, then under the effect of the closed loop control system ,increasing Current of arc motor's stator winding on the left side of the rotor to $\Delta i_{k x}(\mathrm{k}=1,2,3)$, turning into $i_{k x}^{\prime}=i_{k}+\Delta i_{k x}(k=1,2,3)$ and decreasing Current of arc motor's stator winding on the right side of the rotor to $\Delta i_{k x}(k=1,2,3)$, turning into $i_{k x}^{\prime}=i_{k}-\Delta i_{k x}(k=1,2$, $3)$, under the differential action of electromagnetic magnetic pull(which is generated by 2 parts of arc motor's winding), making the rotor return to the center and balance position of the stator, similarly, we can also analysis the Working principle of Rotor suspension in the direction of $y$ axis. Stator winding of arc motor are arranged symmetrically around the rotor, and connect the same 3 phase alternating-current In parallel, 4 parts of arc motor's stator winding generate complex rotating magnetic field under the effect of 3 phase alternating-current, then jointly driving the rotor to rotate after making the stator stable, make Rotor suspension stable, Fig. 2 is 4 pole complex rotating flux map produced by 4 parts of arc motor's winding.
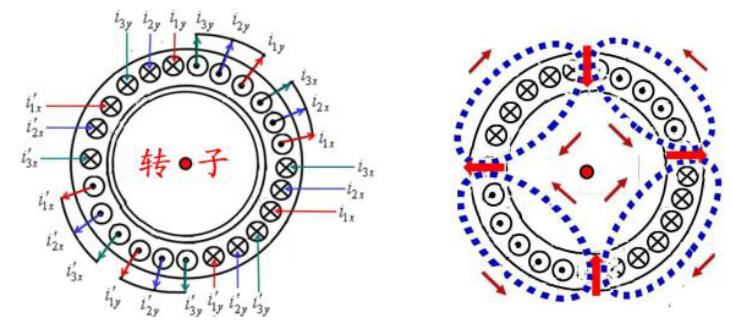

Figure 1. Electrifying State of AC Magnetic Motor

Figure 2. Rotating Magnetic Field of AC Magnetic Motor

\section{Suspension and rotating model of ac magnetic levitation induction motor}

\subsection{The change rule of air gap length between the stator and rotor}

If the rotor is pillar, fig. 3 is the sketch map when rotor displacement occurred, the air gap length $\delta(\theta)$ between rotor outside surface and stator internal surface could be described as an eccentric distance of the rotor in the $x$ and $y$ direction. Assume unilateral average air gap length is $g_{0}=R-r$ and the rotor offset along some direction $\left(\theta_{0}\right)$ is $\rho$. As shown in Fig. 3, we can know that $\rho \cos \left(\theta-\theta_{0}\right)+r+\delta(\theta)=R$, hence, we obtain the air gap length of optional position in space

$$
\delta(\theta)=g_{0}-(x \cos \theta+x \sin \theta)
$$

Where $\theta$ is spatial vector angle, $x$ and $y$ are the rotor displacement along $x$ and $y$ direction.

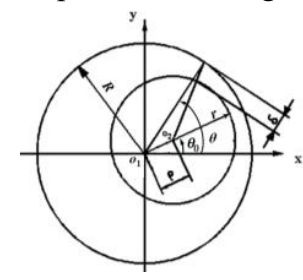

Figure 3. Sketch Map of Air Gap

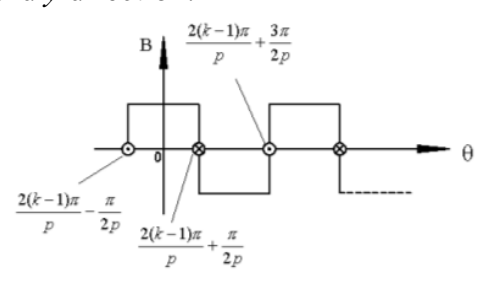
Magnetic Diagram of Rotor
Figure 4. Distribution Curve of

\subsection{Distribution regularities of magnetic induc- tion intensity of motor air gap}

According to magnetic field theory, rotating magnetic induction intensity of single-phase winding of $P$ antipode pole appear rectangular distribution on the circumference of air gap effective length $g$ with the action of current $i(t)$ ${ }^{[8]}$, as the Fig. 4 is shown, we can get distribution regularities of air gap magnetic induction intensity 


$$
B(\theta, t)= \begin{cases}\frac{N i(t) \mu_{0}}{2 g} & \frac{2(k-1) \pi}{p}-\frac{\pi}{2 p} \leq \theta<\frac{2(k-1) \pi}{p}+\frac{\pi}{2 p} \\ -\frac{N i(t) \mu_{0}}{2 g} & \frac{2(k-1) \pi}{p}+\frac{\pi}{2 p} \leq \theta \leq \frac{2(k-1) \pi}{p}+\frac{3 \pi}{2 p}\end{cases}
$$

Where $k$ is natural number, $\mu_{0}$ is air permea-bility of magnetic field, $\mu_{0}=4 \pi \times 10^{-7}, N$ is windings number of motor stator winding, $g$ is air gap length between motor stator and rotor.

It is very troublesome to obtain compound magnetic induction intensity produced by three-phase current on air gap circumference, due to air gap magnetic induction intensity is spatial rectangular wave. For convenient analysis, to expand magnetic induction intensity distributed by each rectangular wave to spatial position fundamental wave and higher harmonic through Fourier series, it mean

$$
\begin{gathered}
B(\theta, t)=\left(\frac{N I \mu_{0}}{2 g} \cdot \frac{4}{\pi}\right)\left(\cos p \theta-\frac{1}{3} \cos 3 p \theta+\right. \\
\left.\frac{1}{5} \cos 5 p \theta+\cdots\right) \cos \omega t
\end{gathered}
$$

Four part of arc stator three-phase winding are consist of three single-phase windings, which are symmetrically distributed on stator circumference and generate magnetic induction intensity of pulse vibration respectively. The each phase axis of three-phase winding after the whole motor stator winding compounded is successively separated by $120^{\circ}$ electrical angle in space. The magnetic induction intensity of fundamental wave is successively separated by $120^{\circ}$ electrical angle in space as well after ignoring equation (3) higher harmonic, according to equations (2) and (3) can be obtain

$$
\left\{\begin{array}{l}
B_{A}(\theta, t)=B_{m} \cos p \theta \cos \omega t \\
B_{B}(\theta, t)=B_{m} \cos \left(p \theta-120^{\circ}\right) \cos \left(\omega t-120^{\circ}\right) \\
B_{C}(\theta, t)=B_{m} \cos \left(p \theta-240^{\circ}\right) \cos \left(\omega t-240^{\circ}\right)
\end{array}\right.
$$

Where $B_{m}$ is magnetic induction intensity amplitu-de can be written as

$$
B_{m}=\frac{2 \mu_{0} N I}{\pi g}
$$

The size of $B_{m}$ depends on current winding inputted and air gap length. The three-phase fundamental wave synthesis of magnetic induction intensity generated by the whole stator three-phase winding magnetic potential between stator and rotor magnetic field air gap can be determined from equation (5), we obtain

$$
B_{s}(\theta, t)=B_{m} \cos (\omega t-p \theta)
$$

Where $\theta$ is tangential angle that wound stator axis, $P$ is number of pole pairs of magnetic field generated by motor winding.

The flux of stator winding of magnetic levitation induction motor rotatable cut armature winding in the space and response to AC electromotive force and current in rotor. Similarly, the rotating magnetic flux can be generated in air gap when alternating current in the rotor, where magnetic induction intensity can be written as

$$
B_{r}(\theta, t)=\beta B_{m} \cos (\omega t-p \theta-\alpha)
$$

Where $\beta$ is induction coefficient of motor stator and rotor winding, $\alpha$ is phase difference between rotating magnetic field of stator winding and rotating magnetic field of rotor winding including displacement of rotor rotation angle, $\beta$ and $\alpha$ all depend on stator and rotor structure and slip ratio of magnetic levitation induction motor.

The total magnetic induction intensity generated by stator and rotor winding on air gap of circumference can be written as

$$
B(\theta, t)=B_{m}(\cos (\omega t-p \theta)+\beta \cos (\omega t-p \theta-\alpha))
$$

As the equation (8) is shown, the distribution regularities of magnetic induction intensity of magnetic levitation induction motor air gap is related to number of pole pairs of magnetic field generated by stator winding, angular position that wound stator axis and phase difference between stator and rotor rotating magnetic field.

\section{Analysis of finite element simulation of ac arc magnetic levitation induction motor}

Table 1. Model parameters of ac arc magnetic levitation induction motor

\begin{tabular}{cc}
\hline Parameter name & Parameter values \\
\hline Inner diameter of stator & $64.5 \mathrm{~mm}$ \\
Outer diameter of stator & $75 \mathrm{~mm}$ \\
Tooth width & $14 \mathrm{~mm}$ \\
Air-gap length & $0.25 \mathrm{~mm}$ \\
Axial length & $20 \mathrm{~mm}$ \\
Numbers of winding ( A set of three phase & 130 \\
winding) & \\
\hline
\end{tabular}

Structural parameters of the arc magnetic levitation induction motor are shown in Table 1. Geometric models of rotor and stator of the arc magnetic levitation induction motor are established and meshed according to the parameters in Table 1. Fig. 5 is enlarged view of air gap grids. After finite element calculation, distribution of magnetic field lines of the initial moment can be got as shown in Fig. 6. 


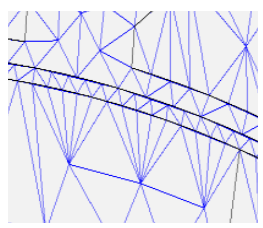

Figure 5. Air Gap Meshing

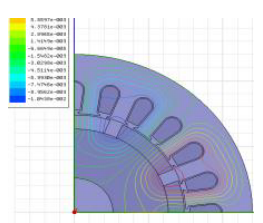

Figure 6. Magnetic Field Lines
Fig. 7 shows the distribution of the magnetic flux density cloud images at the motor's initial moment. Fig. 8 is a magnetic flux density distribution after the operation of a rotation period. Magnetic induction in the middle of stator, rotor magnetic circuit and air-gap is distributed more evenly, the magnetic flux density is larger in the tooth of the stator, at the edge of shaft and the stator periphery occur a certain magnetic leakage, but not obvious.
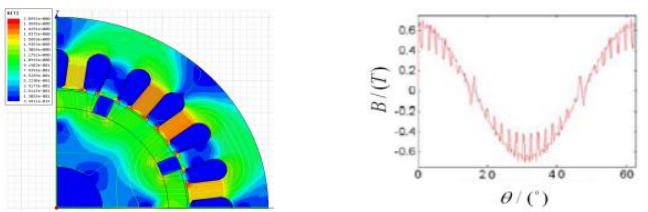

Figure 7 Magnetic Flux Density Figure 8 Magnetic Flux Change Cloud during Rotating

\section{The conclusion}

1) This paper, based on the traditional motor technology, studied the principle a kind of arc motor and analyzed the basic composition and working principle according to the electromagnetic bearing suspension technology.
2) Established the coordinate change rule of air-gap length and air-gap field distribution model of the arc magnetic levitation induction motor.

3) Based on the air-gap magnetic field distribution, established the model of magnetic suspension force and electromagnetic torque of the motor by the principle virtual displacement.

4) Verified the distribution characteristics of air-gap magnetic induction intensity, magnetic suspension force and electromagnetic torque of the arc magnetic levitation induction motor through finite element analysis.

Thank the China Natural Science Foundation for this project. Item Number: 51375427.Thank the Jiangsu Natural Science Foundation for this project. Item Number: BK20131232. Thank the Jiangsu Innovation Foundation of Enterprises and Universities Research for this project. Item Number: BY2014117-08 and BY2015061-04.

\section{References}

1. A. Chiba, T. Deido, T. Fukao, et al. IEEE Transactions on Energy Conversion. 9(1), 61 (1994)

2. Nian Heng, He Yikang. Proceedings of the CSEE. 23(11), 139 (2003)

3. G. J. Wang, Journal of Mechanical Transmission. 28(4), 1 (2004)

4. J. F. Wu, G. L. Zhou, and P. Fu. Journal of Qingdao Institute of Chemical Technology. 23(3), 54 (2002).

5. W. K. S. Khoo. IEEE Transactions on Magnetics, 41 (4):1289(2005)

6. L. Zeng, and H. Q. Zhu. China Mechanical Engineering,. 4(4), 387 (1999).

7. K. Davey, G. Vachtsevanos, and R. Powers. IEEE Trans.on Magn. 23 (1), 273 (1987)

8. P. Z. Chen, and L. T. Yan. Motor Electromagnetic Field Theory and Calculation. 3 (1986) 